\title{
SIMULATION OF MASKED COMMUNICATION WITH CHAOTIC LASERS
}

\author{
Renato Mariz de Moraes; Luiz de Barros O. Netoł and José Roberto Rios Leite \\ Departamento de Física, Universidade Federal de Pernambuco, \\ 50732-910, Recife PE Brazil \\ E-mail : rios@df.ufpe.br
}

\begin{abstract}
Resumo : Este artigo descreve uma técnica de comunicações baseada empulsos de lasers caóticos. Um sinal portador de informação é camuflado por pulsos portadores caóticos, e recuperados através de sincronização caótica em um receptor caótico. Dois circuitos analógicos, representando lasers caóticos de $\mathrm{CO} 2$, simulam o sistema. A pulsação caótica do primeiro sistema é modulada e transmitida ao segundo sistema, o qual atua como receptor e demodula o sinal por sincronização. Séries temporais fornecem o espectro de potência e mapas da seção de Poincaré do sinal transmitido. Verificamse a aparente ausência da modulação e a natureza camuflada das mensagens enviadas.
\end{abstract}

Abstract:This paper describes communication with chaotic laser pulses. An information bearing signal is masked within the chaotic carrier pulses and recovered via chaos synchronization in a chaotic receiver. Two analog circuits, representing chaotic $\mathrm{CO}_{2}$ lasers, simulate the system. The chaotic pulsation from the first circuit was modulated and transmitted to the second one which behaved like a receptor, demodulating the chaotic signal through synchronism. Temporal series give power spectrum and return maps from Poincaré section of transmitted signal. In these no trace of modulation is observable, which verifies the masked nature of the sent messages.

Keywords: Chaotic lasers, synchronization of chaos, chaotic communication.

\section{INTRODUCTION}

Within this decade completely new techniques of communication exploiting

the properties of classical chaotic systems have been proposed and are under development [1]-[3]. Chaotic communication has a large potential for transmitting information in useful way and, for the first time, the GLOBECOM Conference 1998, reserves a section only for this subject [4]. Various concrete advantages have been theoretically studied but much of the work is at feasibility stage and no commercial implementation of chaos based communication links exists to date.

The two main techniques applying chaos to communications are: synchronized chaotic systems communication [2] and communicating with chaos [3]. This second technique is based on the information theoretic formalism of chaos [5] and takes advantage of the fact that chaotic dynamical system can

\footnotetext{
*Renato M. de Moraes is with Depto. de Telemática, FEEC- UNICAMP. E-mail : rmariz@dt.fee.unicamp.br .

${ }^{\dagger}$ Luiz de Barros O. Neto is with NLINK Ltda. E-mail : luiz@nlink.com.br.
}

be controlled by small perturbation [6]. The first technique uses the singular, and not fully understood, characteristics of synchronization between chaotic systems and is applicable in secure communication analogous to spread spectrum communication [7].

Synchronized chaotic systems communication is represented in Fig. ??. The chaotic transmitter is a chaotic nonlinear generator whose signal contains the complex structure of the chaotic attractor. One way of message masking is to add the message signal to the chaotic carrier signal at the transmitter, producing an overall chaotic signal, as shown in Fig. 1a. At the receiver a second nonlinear system, sensitive to synchronization, extracts the message masked within the chaotic time series of the transmitter. Another procedure, named Chaos Shift Keying or CSK [8] is based on modulatation of one parameter ( digital modulation ) of the nonlinear transmitter, schematized in Fig. 1b. Appropriately choosing two values of the parameter, the transmitter chaotic output contains a binary encoding that will be demodulated by the nonlinear receiver, set to go in and out of synchronism, producing an error signal which after suitable filtering recover the modulation message [2], [8]. For an eavesdropper, without an appropriate synchronizing receiver, a chaotic time series would be picked up, but the message will remain buried in the chaos.

Breaking masked messages transmitted within a chaotic signal, without a synchronizing receiver, is only possible when certain redundancies, contained in the chaotic time series, are violated. For a system involving low dimensional chaotic attractors, having unidimensional Poincaré section and single branch 1-D return map, this message breaking was numerically demonstrated using Lorenz equations [9]. In general, a precise set of theoretical limiting conditions for secure transmission with chaotic signals and synchronizing receivers is not yet established. Therefore the study of experimental transmission and the simulation of concrete models associated to real laser systems is relevant. Here we present the results of analog circuit simulation of CSK communication between two $\mathrm{CO}_{2}$ lasers with saturable absorber. A letter presentation of the main results has been published in [10]. Power spectrum, Poincaré section, first return maps and time return map of the masked chaotic signal were tested and no breaking of the modulation message was possible. The circuits were set up to simulate real $\mathrm{CO}_{2}$ lasers with saturable absorber, whose chaos is fairly well understood [11]. 
(a)

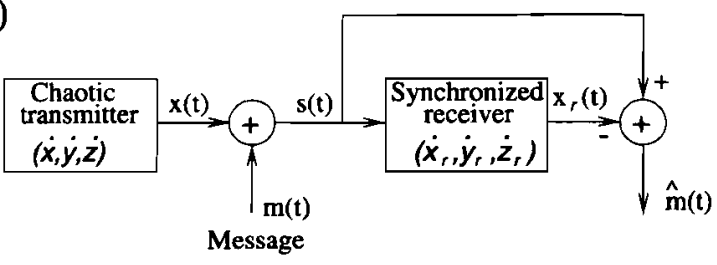

(b)

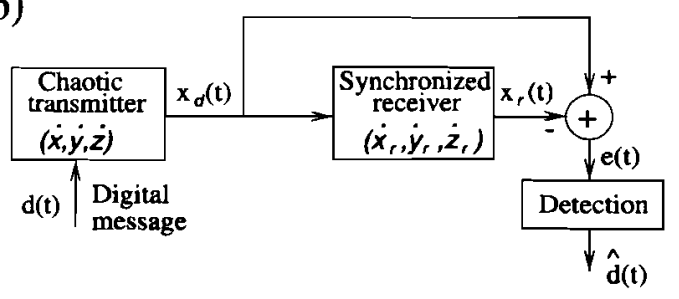

Figure 1: Chaotic communication systems with masked signal. a) The information message $m(t)$ is added to the chaotic carrier $x(t)$ producing an overall chaotic signal $s(t)$. The transmitted message is recovered subtracting the chaotic carrier from the corresponding variable at the receiver. $b$ ) A digital message $d(t)$ modulates a parameter in the chaotic transmitter. At the receiver the error signal $e(t)$ is filtered in the detection block to recover the message.

\section{THE CHAOTIC $\mathrm{CO}_{2}$ LASERS AND THEIR SYNCHRONIZA- TION}

The circuits were set up to simulate real $\mathrm{CO}_{2}$ lasers with an intra cavity saturable absorber cell.

The chaotic pulsation of single mode $\mathrm{CO}_{2}$ laser with an intra cavity saturable absorber (LSA) has been extensively studied [11]. Synchronization of chaotic $\mathrm{CO}_{2}$ lasers has been demonstrated [12], [13]. The circuit implementation given here corresponds to two such lasers in a master-slave coupling configuration. With a low threshold to bifurcate into periodic and chaotic pulsations, associated to passive $Q$-switching, this laser has been a test system to verify general properties of nonlinear dynamical systems [11].

The models introduced to describe the LSA use the rate equations approximation for homogeneously broadened gain and absorbing media.

The light intensity is therefore used as a dynamical variable along with the population differences of the material media inside the cavity. The dynamics of the laser in single mode operation is independent of space variables. Accordingly, the mean intensity $I(t)$, the mean gain medium population inversion $U(t)$, the ground state population depletion of the gain medium, $W(t)$, and the mean population difference of the absorber $\bar{U}(t)$, are the four independent variables describing this laser [11].

The coupling of two such lasers is also described by rate equations without optical frequency locking phenomena [12]. In a master-slave configuration the two coupled lasers are described by eight equations [11], [12]:

$$
\begin{aligned}
& \dot{I}_{T}=I_{T}\left(U_{T}-\bar{U}_{T}-1\right) \\
& \dot{U}_{T}=\epsilon_{T}\left[W_{T}-U_{T}\left(1+I_{T}\right)\right] \\
& \dot{W}_{T}=\epsilon_{T}\left(A_{T}+b_{T} U_{T}-W_{T}\right) \\
& \dot{\bar{U}}_{T}=\bar{\epsilon}_{T}\left[\bar{A}_{T}-\bar{U}_{T}\left(1+a_{T} I_{T}\right)\right] \\
& \dot{I}_{R}=I_{R}\left(U_{R}-\bar{U}_{R}-1\right) \\
& \dot{U}_{R}=\epsilon_{R}\left[W_{R}-U_{R}\left(1+I_{R}\right)\right] \\
& \dot{W}_{R}=\epsilon_{R}\left(A_{R}+b_{R} U_{R}-W_{R}\right) \\
& \dot{\bar{U}}_{R}=\bar{\epsilon}_{R}\left[\bar{A}_{R}-\bar{U}_{R}\left(1+a_{R}\left(I_{R}+c I_{T}\right)\right)\right] .
\end{aligned}
$$

The indices $T$ and $R$ stand for transmitter (master) and receiver (slave), respectively. The parameters in the equations are the normalized relaxation rates, saturation coefficients, and pumping rates in the absorber and amplifiers. Detailed explanation of these dimensionless coefficients and the physical behaviour contained in the above equations for a single laser are given in Ref. [11]. Two relevant parameters are c, the coupling coefficient, and $A_{T}$ the emitter laser pumping rate. It was this rate that was modulated in the communication scheme. A block diagram corresponding to this implementation is shown in Fig. ??. The term $d(t)$ is the digital message, to be transmitted, which modulates the parameter $A_{T}$ in Eq. (3), making it assumes two different discrete levels.

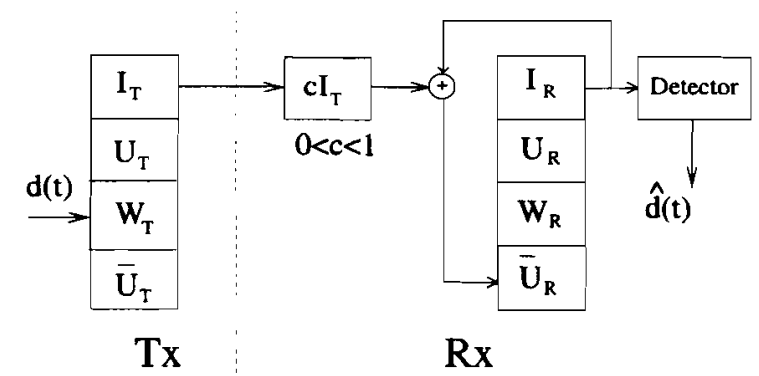

Figure 2: Block diagram of laser communication system. Tx stands for transmitter, and $\mathbf{R x}$ for receiver.

This block diagram should be contrasted with Fig. ??. Here the synchronization is obtained adding a fraction of the transmitted signal, $I_{T}$, to the receiver according Eq. (8). Previous schemes use substitution of variables [2], [8].

The numerical solutions of Eqs. (1)-(4) give rise to periodic and chaotic Q-switching pulsations, with most of the dynamical characteristics observed experimentally [11]. The laser pulses show cascades of period doubling and tangent bifurcations to type I intermittence chaos as the system approaches homoclinic tangency to an unstable periodic cycle. Near the homoclinic tangency the chaotic pulses have a complex structure (Shil'nikov type) consisting of a big spike and a variable number of undulations corresponding to the trajectories spiraling around the unstable limit cycle [11]. 
The return maps [14] reconstructed from this chaotic behavior is 1-D but with a multibranched structure that accumulates to an infinity number as one approaches the homoclinic tangency [11]. This was the region of chaos chosen to generate the modulated signal, as described further.

\section{ELECTRONIC CIRCUITS}

The circuit implementation of equations (1)-(4) is shown in Fig. 3. The operational amplifiers were LF351 and the associated sub-circuits perform the addition, subtraction and integration operations. The $X$ multipliers had a combination of an analog multiplier $A D 734 B Q$ with an operational amplifier, and they implement the nonlinear terms of equation. The operational amplifiers associated with $R_{32}$ and $R_{33}$ work as voltage buffers. If standard node analysis techniques are applied to the circuit given in Fig. 3 a set of equations result which govern the dynamical behaviour of the circuit. So we had

$$
\begin{aligned}
& \dot{I}=\frac{R_{6}}{R_{7} C_{1}}\left[\frac{I U}{R_{2}}-\frac{\frac{1}{R_{1}}+\frac{1}{R_{2}}+\frac{1}{R_{\mathrm{fi}}}}{\frac{1}{R_{3}}+\frac{1}{R_{1}}+\frac{1}{R_{5}}}\left(\frac{I}{R_{3}}+\frac{I}{R_{4}}\right)\right] \\
& \dot{U}=\frac{R_{13}}{R_{14} C_{2}}\left[\frac{W}{R_{9}}-\frac{\frac{1}{R_{*}}+\frac{1}{R_{93}}+\frac{1}{R_{133}}}{\frac{1}{R_{10}}+\frac{1}{R_{11}}+\frac{1}{R_{1,2}}}\left(\frac{U}{R_{10}}+\frac{I U}{R_{11}}\right)\right] \\
& \dot{W}=\frac{R_{21}}{R_{22} C_{3}}\left[\frac{A}{R_{17}}+\frac{b U}{R_{16}}-\left(\frac{\frac{1}{R_{1,4}}+\frac{1}{R_{17}}+\frac{1}{R_{21}}}{\frac{1}{R_{1 \times}}+\frac{1}{R_{19}}+\frac{1}{R_{20}}}\right) \frac{W}{R_{19}}\right] \\
& \dot{\bar{U}}=\frac{R_{30}}{R_{31} C_{4}}\left\{\frac{\bar{A}}{R_{26}}-\frac{\frac{1}{R_{2,}}+\frac{1}{R_{21,}}+\frac{1}{R_{311}}}{\frac{1}{R_{2 \tau}}+\frac{1}{R_{2 \times}}+\frac{1}{R_{2 !}}}\left[\frac{\bar{U}}{R_{28}}+\right.\right. \\
& \left.\left.\left(1+\frac{R_{23}}{R_{24}}\right) \frac{I \bar{U}}{R_{27}}\right]\right\} \text {. }
\end{aligned}
$$

From these equations result that the time scale can be adjusted replacing the values of the four capacitors $C_{1}, C_{2}, C_{3}$ and $C_{4}$ by a common factor. Furthermore, the parameters $\epsilon$, $\bar{\epsilon}, A, \bar{A}, a$ e $b$ can be independently adjusted by setting the respective resistors $R_{14}$ and $R_{22}, R_{31}, R_{32}, R_{33}, R_{23}$, and $R_{15}$. For example, the voltage corresponding to values of $A$ and $\bar{A}$ could be obtained straightforward adjusting the voltage across $R_{32}$ and $R_{33}$, respectively. These and others circuits elements were set as: $R_{1}, R_{2}, R_{3}, R_{4}, R_{15}, R_{25}, R_{26}, R_{27}$, $R_{28}=100 \mathrm{~K} \Omega ; R_{5}, R_{6}, R_{29}, R_{30}=4.17 \mathrm{~K} \Omega ; R_{8}, R_{9}$, $R_{10}, R_{11}, R_{16}, R_{17}, R_{18}, R_{19}=200 K \Omega ; R_{12}, R_{13}, R_{20}$, $R_{21}=7.68 \mathrm{~K} \Omega ; R_{14}, R_{22}=11.7 \mathrm{~K} \Omega ; R_{23}=110 \mathrm{~K} \Omega$; $R_{24}=34.8 K \Omega ; R_{31}=3.48 K \Omega ; R_{32}, R_{33}=1 K \Omega ; R_{34}$, $R_{35}, R_{36}, R_{37}, R_{38}=10 K \Omega ; R_{39}=100 K \Omega ; C_{1}, C_{2}, C_{3}$, $C_{4}=1000 p F$. Using these values the resulting parameters were $\epsilon=0.137, \bar{\epsilon}=1.2, A=1.4$ to $2.1, \bar{A}=2.16, a=4.17$ and $b=0.85$, and the set of equations (9) are equivalent to (1)-(4) after rescaling time by factor of 23990.

It is important to remind that careful offset adjustments had be done to all operational amplifiers, or else intolerable errors would appear in the equations.

The receiver circuit is very similar to the transmitter one, as it is given in Fig. 4. The difference is the inclusion of one more $\mathrm{X}$ multiplier and two other operational amplifier and resistors to allow $I_{T}$ to be injected in the receiver according to

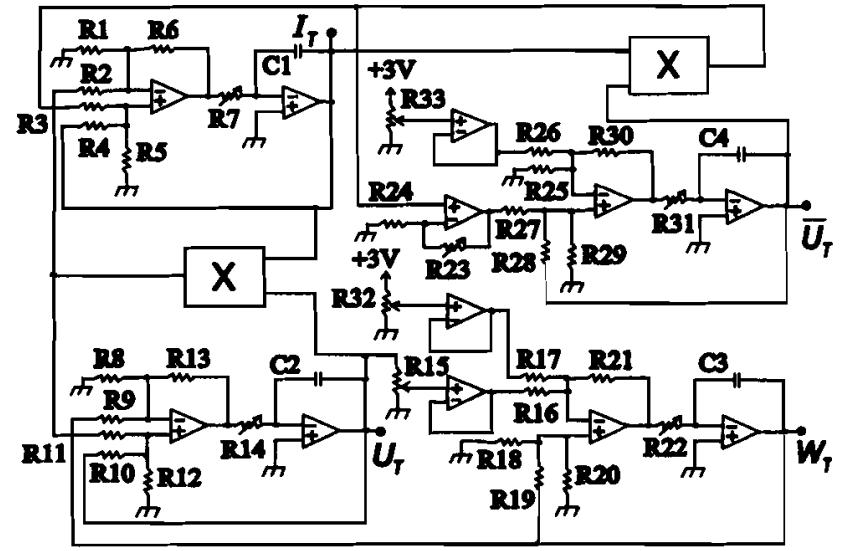

Figure 3: Electronic circuit corresponding to laser equations transmitter.

Eq. (8), and the coupling parameter $c$ which can be adjusted by setting the resistor $R_{39}$ in the circuit.

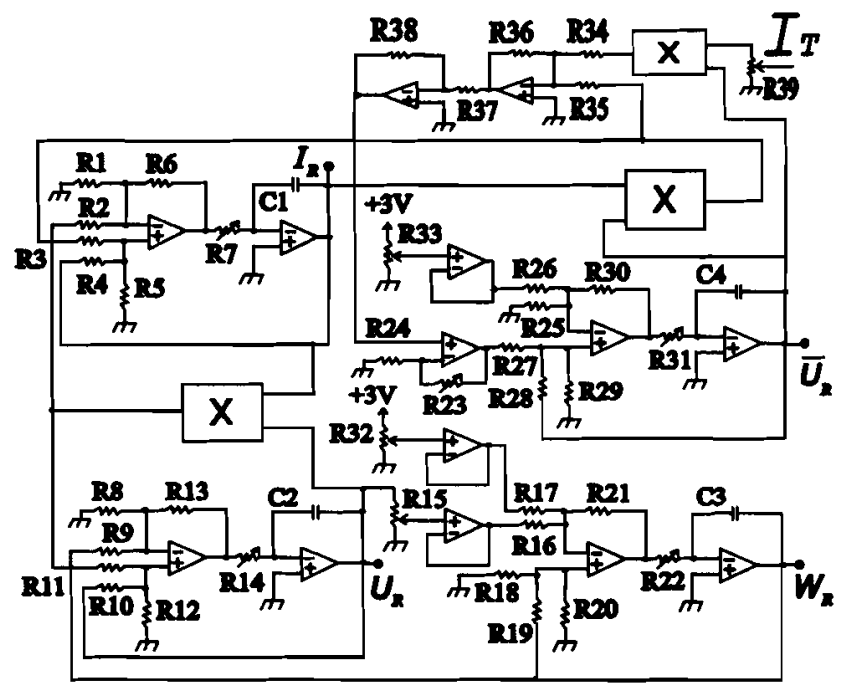

Figure 4: Electronic circuit corresponding to laser equations receiver. Input from the transmitter is represented on the right top of the circuit.

\section{COMMUNICATION}

The output voltage of the transmitter circuit, corresponding to the variable $I(t)$ was the transmitted signal. The receiver circuit was fed by approximately $9 \%$ of the transmitter signal $(c=0.09$ in Eq. (8)). It had a $Q$ switching pulsation going in and out of partial synchronism [12] according to the modulation of the transmitter system. To study the properties of the link, transmitter and receiver signals were simultaneously acquired with 12 bits A/D converters. The modulation bits were also registered during data acquisition, providing complete monitoring of the simulation process, as shown in Figs. 5 and 6.

The typical pulses of the transmitter circuit, are shown in Fig. 5b. They reproduce the numerical solution of Eqs. (1)(4) which by its turn describe the dynamics of the real $\mathrm{CO}_{2}$ 

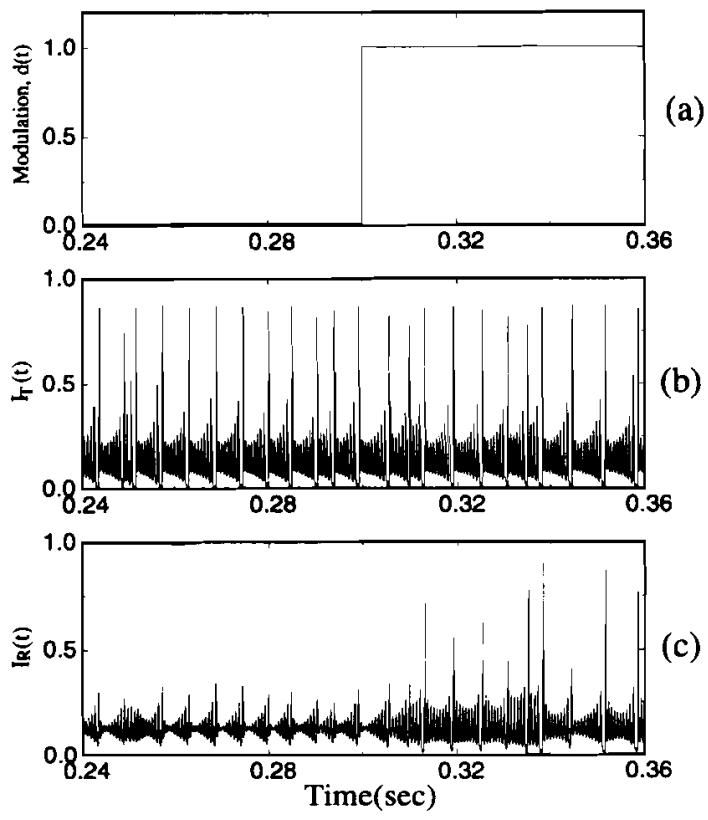

Figure 5: Segments of $a$ ) the digital modulation message, $b$ ) the transmitter output pulses and $c$ ) the receiver pulses, for the two circuits representing chaotic lasers. The modulation of the transmitter gain parameter creates the conditions of bit zero, when the receiver circuit no synchronizes, and of bit one when it synchronizes, with the incoming pulses.

lasers.

To describe the nonlinear dynamics, the notation used in [11] is followed here. A $P^{(n)}$ regime corresponds to a periodic $Q$-switching pulsation with a spike pulse followed by $n$ undulations. $C^{(n)}$ is an intermittent chaotic regime appearing between $P^{(n)}$ and $P^{(n+1)}$ regimes where the system hesitates with pulses having $n+1, n, n-1$, etc, undulations. The depth of modulation used in the pump coefficient $A_{T}$ was $0.4 \%$ and the pulses were always near the $C^{(9)}$ region. Naturally, we could not determine a single $C^{(n)}$ region for each modulation bit for if this were the case the masking would be easily broken. This small depth of modulation must be contrasted to the one used in previous works with the Lorenz circuits and equations [2] that had $10 \%$ modulation and therefore could be broken [9].

To synchronize the receiver laser circuit it was necessary to set its parameters equal to those of the transmitter, except for $A$ and $\bar{A}$, which were set by trial procedure.

The values of parameters used to give Figs. 5 and 6 were established by a manual tuning of the circuits. The receiver parameters were: $\epsilon_{R}=0.137, \bar{\epsilon}_{R}=1.60, A_{H}=2.040$, $\bar{A}_{R}=2.51, a_{R}=4.17, b_{R}=0.85$ and $c=0.09$. The transmitter was set with $\epsilon_{T}=0.137, \bar{\epsilon}_{T}=1.60, \bar{A}_{T}=2.97$, $a_{T}=4.17, b_{T}=0.85$ and the pump modulation for bit zero $A_{T 0}=2.532$ and for bit one $A_{T 1}=2.541$. The temporal duration of the modulation bits was $100 \mathrm{msec}$ which corresponds to approximately 150 periods of the unstable limit cycle that organized the chaotic trajectories of the two cir126 cuits. In the system analysis, the data acquisition rate was $100 \mathrm{~K} \mathrm{~Hz}$, corresponding to 66 points per small cycle.

Figure 6 shows long series of the chaotic transmitted signal and the associated receiver behaviour.
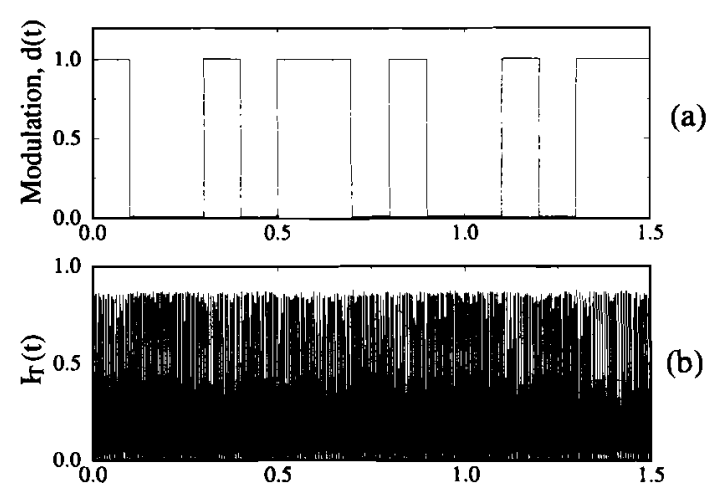

(a)

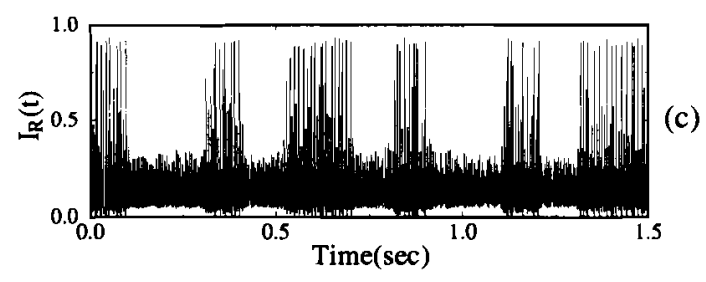

Figure 6: Long segments of $a$ ) the digital modulation message, $b$ ) the modulated transmitter signal and $c$ ) the receiver signal showing the on-off synchronization detection.

Even without performing a systematic study we have verified that for the masked communication scheme to work only partial synchronization was necessary. The big spikes of the receiver pulses, associated to the reinjection portion of the chaotic trajectory, did synchronize with the emitter pulses for the modulation at bit 1 while no synchronization existed when the modulation was slightly shifted (bit 0 ). An electronic signal envelope detector made no error extracting the modulation message from long sequences, from $I_{R}$, as indicated in Figs. ?? and 6c. To this moment we have no physical explanation for the cause of such sensitivity of the receiver system to the reinjection pulses in the specific case of the rate equations of coupled $Q$-switching $\mathrm{CO}_{2}$ lasers.

The power spectrum of the transmitted signals were typical of a broadband chaotic source and no distinction could be made between series with bit 1 or 0 , as seen in Fig. 7 .

To further verify the masking, time series of segments with pure bit 0 and pure bit 1 were also used to reconstruct the phase space of the transmitter with time delayed variables [11].

The highly dissipative chaos permits a three dimensional space reconstruction, with elimination of the $\bar{U}_{T}$ variable. From these reconstructed attractors the Poincaré sections shown in Fig. 8 were extracted. The sections appear approximately as line segments [11]. The relevant aspect is that no distinction appear for the location of the Poincare sections with respect to the modulation condition. At the same time 


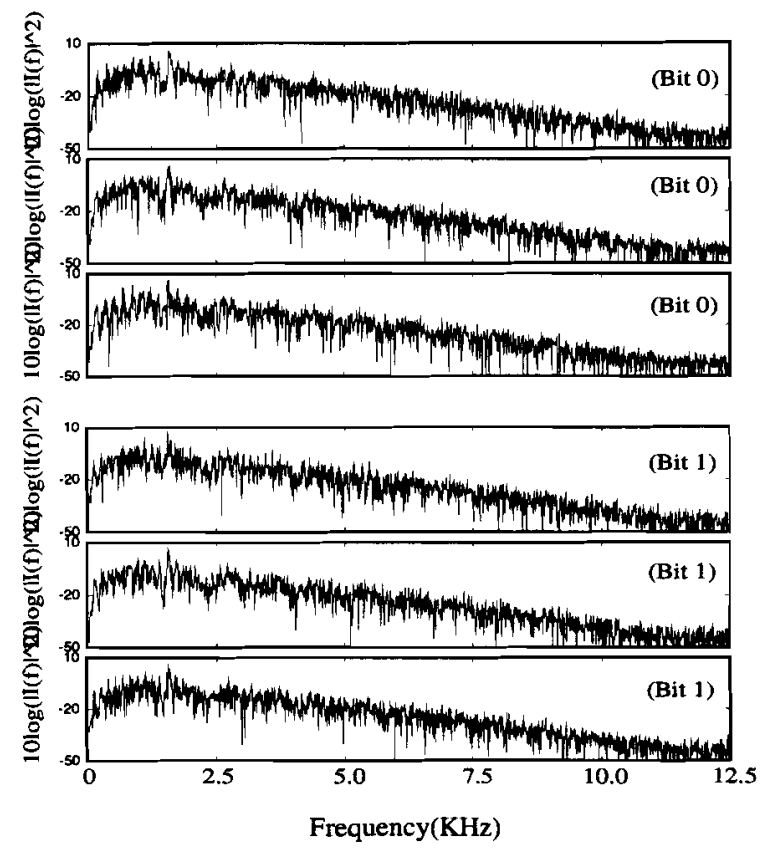

Figure 7: Power spectrum corresponding to three bitO and three bit 1 of transmitted signal. No significant difference is detected.

the synchronizing receiver was clearly detecting the signal message. Similar to the Poincaré sections, indistinguishable results occur for the first return maps and the time return maps constructed from the modulated signal data, as it is shown in Fig. 9. The return maps should appear with the points on eight line segments belonging to the 1-D map of a $C^{(9)}$ chaotic regime [11]. Instead, broad distributions appear, with a significant spread due to the short number of reinjection pulses occurred during parameter modulation which disable a good construction of these maps. The branches for different values of the modulation parameter are badly formed, preventing the distinction between modulation bits.

\section{CONCLUSIONS}

In conclusion we have demonstrated, with analog circuits implementing the rate equations, that lasers with saturable absorber can be used for masked communication using a modulated chaotic signal and a synchronizing receiver. The nature of the chaotic attractor (of the Shil'nikov type here) may keep the signal masked because the signature of change in the modulated chaotic series, given by changes on the 1-D return maps, could not be resolved with multibranched maps typical of homoclinic chaos. The small depth of the modulation parameter and short number of reinjection pulses make difficult the extracting messages by an eavesdropper, while preserving a good synchronization sensibility.

Detailed studies of the noise dependence [15] and the relation with other communication schemes using non-chaotic

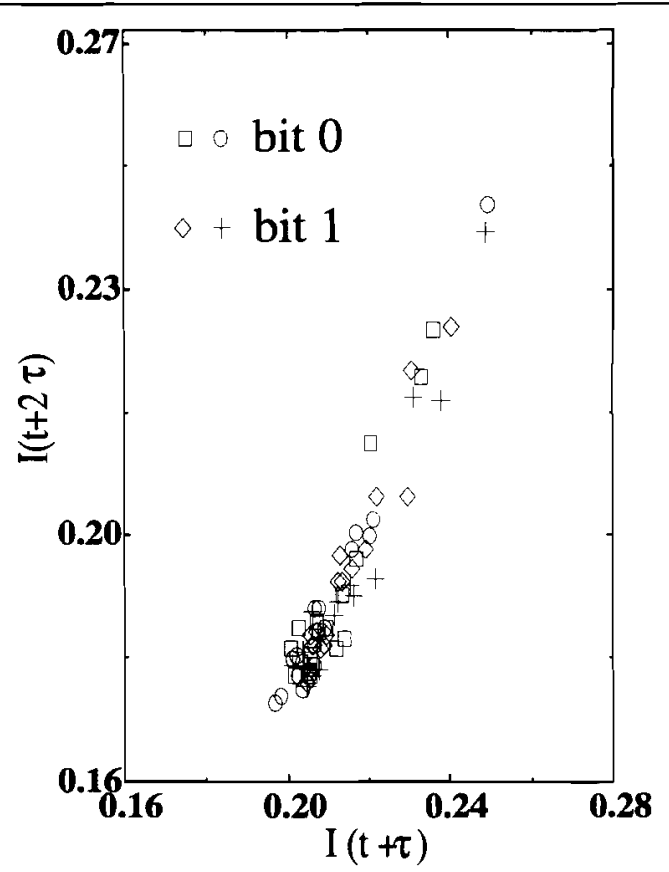

Figure 8: Poincaré section of the reconstructed attractors when the transmitter laser is modulated and transmitting as in Fig. 6. No distinction is possible between bits 0 and 1 .

lasers remain to be done [16]. The synchronization and nonsynchronization between $\mathrm{CO}_{2}$ lasers has no precise explanation and is an active research subject [17] that can be exploited by electronic simulation.

The circuits, as analog computers in which one can easily change parameters, might also be used to test other recently proposed schemes of control of chaos [18] and synchronization of chaotic systems [19], [20]. The same equations and circuits presented here have also been used to study the grammar of the chaotic source and to the proposal of compatible coding for pulse sequences of this laser to make them useful as optimized information channel [21].

\section{ACKNOWLEDGMENTS}

The authors acknowledge Hugo L. D. de S. Cavalcante for his contributions to the final stage of this work and the support from Conselho Nacional de Desenvolvimento Científico e Tecnológico (CNPq) and Fundação de Amparo à Ciência e Tecnologia de Pernambuco (FACEPE), Brazil.

\section{REFERENCES}

[1] L. M. Pecora, and T. L. Carrol, "Synchronization in chaotic systems", Phys. Rev. Lett. 64, pp. 821-824, 1990.

[2] K. M. Cuomo, A. V. Oppenheim, and S. H. Strogatz, "Synchronization of Lorenz-based chaotic circuits with applications to communications", IEEE Trans. on circ. and syst. II 40 (10), pp. 626-633, 1993. 

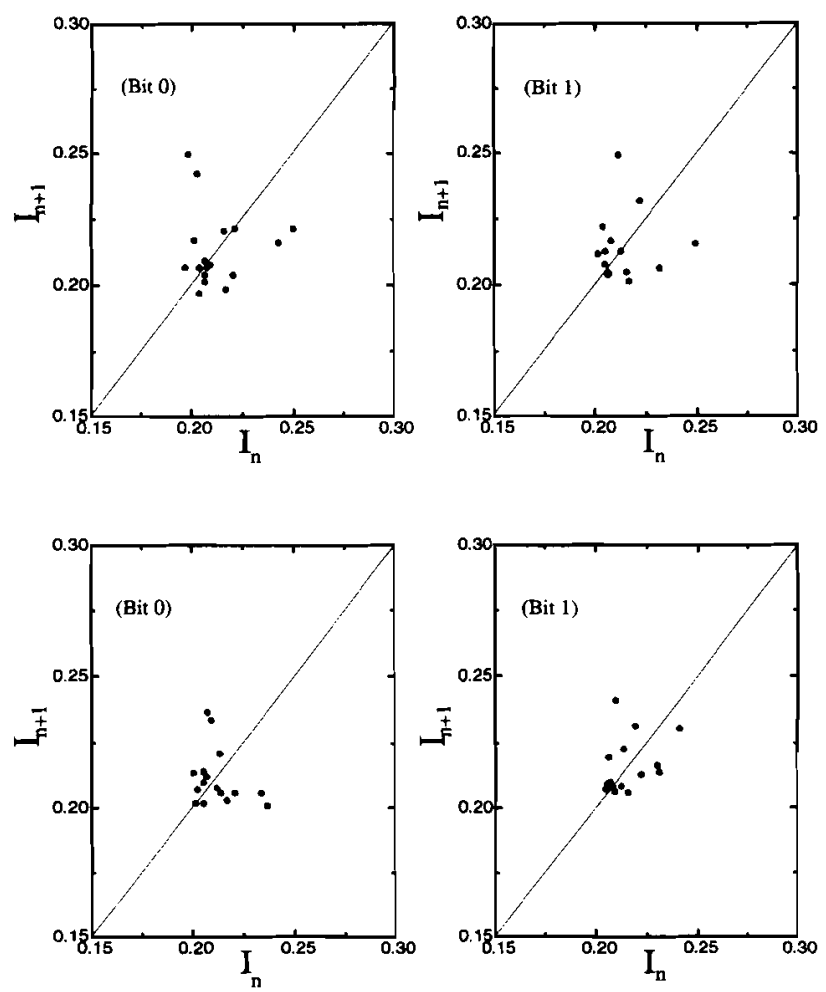

Figure 9: Time return maps of two bit 0 and two bit 1 of transmitted signal, to be compared.

[3] S. Hayes, C. Grebogi, and E. Ott, "Communicating with chaos”, Phys. Rev. Lett. 70, pp. 3031-3034, 1993.

[4] IEEE Global Telecommunications Conference, 8-12 November 1998, Sydney Australia.

[5] R. Shaw, "Strange attractors, chaotic behaviour, and information flow", Z. Naturforsch. 36a, pp. 80-112, 1981.

[6] E. Ott, C. Grebogi, and J. A. Yorke, "Controlling chaos”, Phys. Rev. Lett. 64, pp. 1196-1199, 1990.

[7] K. S. Halle, C. -W. Wu, M. Itoh, and L. O. Chua, "Spread spectrum communication through modulation of chaos", Int. J. Bif. Chaos, vol. 3, No. 2, pp. 469-477, 1993.

[8] H. Dedieu, M. P. Kennedy, and M. Hasler, "Chaos shift keying: modulation and demodulation of a chaotic carrier using self-synchronizing Chua's circuits", IEEE Trans. on circ. and syst. II 40 (10), pp. 634-642, 1993.

[9] G. Pérez, and H. A. Cerdeira, "Extracting messages masked by chaos”, Phys. Rev. Lett. 74, pp. 1970-1973, 1995.

[10] Renato M. de Moraes, L. de B. Oliveira-Neto, and J. R. Rios Leite, "Analog circuits simulation of communication with chaotic lasers", Appl. Phys. Lett., 70 (11), pp. 1357-1359, March 1997.

[11] M. Lefranc, D. Hennequin, and D. Dangoisse, "Homoclinic chaos in a laser containing a saturable absorber", J. Opt. Soc. Am. B. 8 (2), pp. 239-249, 1991.
[12] Y. Liu, P. C. de Oliveira, M. B. Danailov, and J. R. Rios Leite, "Chaotic and periodic passive Q-switching in coupled $\mathrm{CO}_{2}$ lasers with a saturable absorber", Phys. Rev. A 50, pp. 3464-3470, 1994.

[13] T. Sugawara, M. Tashikawa, T. Tsukamoto, and T. Shimizu, "Observation of synchronization in laser chaos”, Phys. Rev. Lett. 72, pp. 3502-3505, 1994.

[14] See, for instance, to take an idea on how to construct such maps: T. Braun, and J. A. Lisboa, "Characterization of homoclinic chaos in a glow discharge through return maps", Int. J. Bif. Chaos, vol. 4, No 6, pp. 14831493, 1994.

[15] E. Rosa Jr., S. Hayes, and C. Grebogi, "Noise filtering in communication with chaos", Phys. Rev. Lett. 78, pp. 1247-1250, 1997.

[16] C.-S. Zhou, and T.-L. Chen, "Robust Communication via synchronization between non-chaotic strange attractors", Europhys. Lett. 38, pp. 261-265, 1997.

[17] T. Tsukamoto, M. Tachikawa, T. Hirano, T. Kuga, and T.Shimizu, "Synchronization of a laser system to a modulation signal artificially constructed from a strange attractor”, Phys. Rev. E 56, pp. 6564-6568, 1997.

[18] Y.- C. Lai, and C. Grebogi, "Synchronization of chaotic trajectories using control", Phys. Rev. E 47, pp. 2357 $2360,1993$.

[19] M. Ding, and E. Ott, "Enhancing synchronism of chaotic systems", Phys. Rev. E 49, pp. R945-R948, 1994.

[20] N. F. Rulkov, M. M. Sushchick, L. S. Tsimring, and H. D. I. Abarbanell, "Generalized synchronization of chaos in directionally coupled chaotic systems", Phys. Rev. E 51, pp. 980-994, 1995.

[21] Renato M. de Moraes, "Codificação e capacidade de canal de um laser caótico", Master Thesis, FEECUNICAMP, 1998.

Renato Mariz de Moraes received the Bachelor of electrical engineering degree in 1996 from Universidade Federal de Pernambuco, Recife. He is currently working towards the Master degree at the Universidade Estadual de Campinas (Unicamp), Campinas, Brazil. His research interests include chaos, coding, and digital communication.

Luiz de Barros O. Neto received the Bachelor of electrical engineering degree in 1995 from Universidade Federal de Pernambuco, Recife, Brazil. Currently, he is an Internet provider manager at NLINK Ltda., Recife, Brazil. His research interests are computer networks and programming languages.

José Roberto Rios Leite received the Bachelor of electrical engineering degree in 1968 from Universidade Federal de Pernambuco, Recife, Master degree in Physics in 1972 from Pontifícia Universidade Católica do Rio de Janeiro, Brazil, and Ph.D. degree in Physics in 1977 from Massachussets Institute of Technology, USA. His research subjects include laser spectroscopy, nonlinear optics and chaos. 